\title{
LES GUESDISTES, LA DEUXIEME EGALITE ET LA COMMUNE (I880)
}

Comment le mouvement socialiste français renaissant a-t-il vu la Commune? Qu'en a-t-il retenu? La Commune est-elle un modèle, un mythe ou une étape de la lutte vers la Révolution? Nous avons choisi la deuxième série du journal guesdiste L'Egalité, publiée du 21 janvier 1880 au 25 août 1880: "Pour la première fois en France, un <journal ouvrier> au plein sens du mot», comme l'écrit Marx à Sorge en novembre 1880 . Cet hebdomadaire doctrinal paraît après le congrès de Marseille d'octobre 1879, lors de la phase préparatoire à l'adoption d'un programme socialiste commun. Quelle place la Commune a-t-elle tenue lors de cette élaboration?

En 1880, se déroulent les premières manifestations commémorant la Commune. Quelle a été la part ici prise par l'Egalité et quels ont été les thèmes retenus? Etait-ce commémoration seulement quasi religieuse, ou bien recherche d'enseignements qui pourraient servir d'arguments dans la bataille à mener? En 1880 aussi, le 11 juillet, le Parlement votait l'amnistie définitive des Communards. Les raisons en sont claires: faire des concessions suffisantes au peuple pour ne pas laisser au mouvement ouvrier naissant l'occasion de reprendre trop de vigueur.

L'Egalité compte parmi ses collaborateurs d'anciens Communards ou des militants qui avaient joué un rôle en province en 1871: Brousse et Guesde avaient participé à Montpellier à la publication des Droits de l'Homme; Lafargue a joué un rôle important à Bordeaux en 1871. Et puis Malon, J.-B. Clément (correspondant pour l'Angleterre), Frankel (correspondant pour la Hongrie) avaient été membres de la Commune; Eugène Dupont (correspondant pour les Etats-Unis, responsable des questions françaises au Conseil général de l'A.I.T.) avait suivi de Manchester les événements de Paris. Les autres collaborateurs n'ont pas été mêlés aux événements: Fournière, Brugnot, Deville, Ferroul, Labusquière, Marouck, Massard...

Nous nous proposons d'examiner comment l'Egalité a commémoré, défini, interprété la Commune et quels enseignements elle en a retenus pour la réalisation d'un programme collectiviste. Dans les 33 numéros 
de l'hebdomadaire du 2 mars au 30 juin 1880, date à laquelle est publié le projet de programme électoral des travailleurs socialistes, la Commune est présente dans le journal, par allusions ou par évocations. Enfin, le 7 juillet et le 11 août 1880, l'expérience de la Commune est directement invoquée.

\section{La commémoration de la Commune}

Il arrive que l'Egalité commémore directement la Commune. Témoin cette annonce du 10 mars 1880: "A l'occasion du Neuvième anniversaire du 18 mars 1871, l'Egalité publiera mercredi prochain un numéro extraordinaire illustré à l'encre rouge.» Et en effet, le 18 mars, paraît l'Egalité, rouge, dédiée "aux morts anonymes de mars, avril et mai 1871». Devant le succès de cette présentation, les numéros jusqu'au 23 mai seront entièrement imprimés en rouge. Le 23-26 mai, le titre seulement est rouge, tandis qu'une bande noire entoure la première page. Le 11 février, l'Egalité publiait pour la première fois, en huitième page, des gravures. Du 18 mars au 23 mai, celles-ci sont remplacées par des portraits de Communards: Varlin, Flourens, Rigault, Delescluze, Millière, Ferré, Vermorel, Dombrowski, Tony-Moilin et une fresque: "une rue de Paris pendant les journées de mai». Le 23 mai: «Notre dessin, Les Fusillés, bien que déjà paru en 1871, en plein état de siège, a été interdit au dernier moment par la censure de la République-Grévy." Le 18 mars, le récit de la mort de ces Communards, à travers les articles de journaux de l'époque, invite au respect. Face au nom de ces morts, à deux reprises, la liste de leurs vainqueurs, chefs militaires, juges des différents conseils de guerre. A partir du 31 mars, des «éphémérides communeuses» rappellent les grandes dates de la Commune. Le 23 mai, un long article tente d'expliquer les réactions de la province. Puis deux manifestations de grande ampleur. Le 17 mars, à SaintMandé, l'organisation d'un banquet commémoratif: trois cents personnes $\mathrm{y}$ participent, des organisations provinciales et étrangères ont expédié des messages que publie le journal; mais, l'Egalité du 24 mars ne rapporte pas le contenu des discours, notamment ceux de Labusquière, Bouis et Guesde. Le 5 mai, dans la rubrique "Le mouvement social en France», paraît l'appel suivant:

"Les groupes: Comité central d'aide aux amnistiés et non amnistiés, Union syndicale des travailleurs de la Seine, Union fédérative (région du Centre), la Fédération des groupes collectivistes et communistes, le Cercle des Egaux, les journaux Le Prolétaire, L'Egalité, réunis pour rendre hommage aux victimes de mars, avril, mai 71 ,

Considérant que le prolétariat doit affirmer sa scission complète avec la bourgeoisie, 
Se doit à lui-même d'honorer ceux qui sont morts pour son émancipation sociale en 1871

Nous estimons que les travailleurs, en honorant leurs morts, honorent les principes qui les ont guidés dans leurs revendications sociales et doivent guider le parti socialiste ouvrier dans l'avènement de son affranchissement."

On projette le 23 mai une manifestation de la Bastille au PèreLachaise; l'Egalité ne figure pas parmi les premiers appelants, mais s'y associe. Aussitôt, l'opinion se déchaîne. Le 12 mai, l'Egalité dénonce le gouvernement, qui a fait savoir dans le Journal Officiel que "Quelques journaux s'efforcent de provoquer les citoyens à une manifestation sur la voie publique pour la fin du mois de mai. Ces provocations tombent sous le coup de la loi." Puis le J.O. reproduit le texte de la loi des 7-9 juin 1848. L'Egalité compare alors la situation française à celle de Russie, ou à ce qu'elle avait été sous le Second Empire et conclut: "Tout ce que nous pouvons dire pour l'instant, c'est que sans la note de l'Officiel, on aurait été 10.000 sur la place de la Bastille à conduire le deuil de nos vaincus. Grâce à la note on sera 50.000. Ce sera le premier - et le seul - résultat de la politique des résultats [sic]."

Le 23 mai, l'Egalité rappelle brièvement le sens de cette manifestation. Un supplément en noir au journal contient le compte rendu de la journée. Vingt-cinq mille manifestants ont accompli «jusqu'au bout et malgré tout, [ce qu'ils] considéraient comme le plus saint des devoirs". La police quadrillait les lieux et intervint contre toute tentative de défilé. Quelques manifestants parvinrent à jeter des immortelles dans la fosse commune du Père-Lachaise. Ainsi donc, journée agitée, mais finalement, les forces de l'ordre n'ont pas été débordées. On ne peut parler d'échec en raison de l'isolement des groupes organisateurs, et ceci d'autant plus que c'était la première tentative sérieuse de commémoration sur ces lieux de la répression communarde. L'Egalité est menacée de poursuites pour "délit d'excitation à la haine et au mépris des citoyens les uns contre les autres». Rien ne viendra finalement.

\section{Les interprétations et la représentation de la Commune}

Dans cette courte période, les collaborateurs de l'Egalité donnent une définition de la Commune dont ils tentent naturellement de tirer des enseignements.

Peu de place relativement est accordée à la répression. Le 2 mars, sous le titre, "Pas d'amnistie», un montage d'articles du Gaulois, du Petit Moniteur, de La Petite Presse et de La Patrie vient rappeler les «exploits dont le parti de l'ordre avait illustré son entrée victorieuse 
dans Paris». Le récit de la mort de Communards, la liste des officiers de l'armée versaillaise, des juges, des membres de la Commission des grâces et des vingt-sept Communards qui ont été exécutés complètent cet aspect. En revanche, un long article de deux pages, le 23 mai, sous le titre, "Les massacres de mai et les radicaux", fait la critique du livre de Camille Pelletan, Questions d'histoire, de l'attitude des radicaux au moment de la Commune et de leur tentative de récupération de la Commune. "Pendant deux mois leur nouveau journal a décrit l'horrible carnage, fouillé les plaies, manié les cervelles, empilé les victimes et battu le rappel de l'abonnement avec les os des fédérés." L'article, dans sa véhémence, tend à prouver que ce sont les radicaux qui ont éteint tout soulèvement provincial, qu'ils ont contribué, par leur «abstention déguisée» à Paris, à aider le gouvernement de Thiers. "Une des causes principales des massacres de Mai fut la trahison ou l'inaction voulue des chefs du radicalisme.» L'attitude de Clemenceau, de Louis Blanc, de Tolain avait déjà été dénoncée le 18 mars.

Plus que sur l'aspect terrible de la Semaine sanglante, les socialistes de l'Egalité veulent insister sur ses enseignements politiques. La Commune est ainsi définie par rapport aux préoccupations politiques du moment: la constitution d'un parti ouvrier avec un programme socialiste.

Pour l'Egalité, la Commune représente une étape importante dans les luttes sociales internationales. Un feuilleton épisodique analyse les mouvements paysans en Allemagne au XVIe siècle. L'étude de la révolte lyonnaise de 1831 lui fait suite et prolonge le court article que 'Egalité du 18 mars 1880 avait consacré aux trois mouvements "précurseurs» de la Commune: Conjuration des Egaux, insurrection de Lyon en 1831, et juin 1848. La Commune est conçue comme héritage et comme point de départ. Elle a été:

«1e) Une Révolution ouvrière continuant et complétant les journées de juin 1848 et les insurrections lyonnaises des premières années du règne de Louis-Philippe.

2e) Une Révolution économique ou sociale dont les racines plongent jusqu'à la fin du siècle dernier, en pleine conjuration des Egaux.

Le 18 Mars est presque exclusivement prolétarien.»

Ici apparaît un élargissement de la notion de "prolétariat». «[...] Si la bourgeoisie s'y trouve représentée, c'est par ses <déclassés>, par ses prolétaires, pourrait-on dire, journalistes et étudiants qui vivent de leur travail et dont l'activité cérébrale, comme l'activité musculaire de la classe ouvrière, est subordonnée dans son exercice et son application à un capital qui est possédé par d'autres." Quel en est le contenu nou- 
veau? "[...] Le 18 Mars est social ou socialiste dans son programme, même dans la partie de ce programme qui paraît au premier abord purement politique. L'autonomie communale [...] n'est [...] qu'un moyen destiné à quoi? A universaliser la propriété. L'outil à l'ouvrier, la terre à celui qui la cultive, lit-on dans une autre proclamation à l'adresse des départements." On évoque les mesures prises par la Commune, tout particulièrement le décret du 16 avril sur les ateliers abandonnés.

"C'est donc bien le droit au capital, le droit à l'instrument et à la matière de la production, et non plus seulement le droit au travail qui a été affirmé - incidemment, il est vrai - en pleine bataille, par la Commune de Paris trop avisée pour confondre 1870 avec 1848 et réduire les revendications ouvrières à la formule rudimentaire et incomplète d'il y a 32 ans.

Mais, c'est surtout dans ses conséquences historiques qui - ne l'oublions pas - plus que le programme et le personnel caractérisent une tentative révolutionnaire, c'est surtout dans son lendemain qui dure encore, dans les espérances et les terreurs qu'il a éveillées d'un bout du monde à l'autre, que le 18 mars a donné sa véritable mesure ouvrière et socialiste [...].

[Les travailleurs dans le monde] fêtent à l'envi la date du 18 mars, comme ouvrant une ère nouvelle, l'ère de leur émancipation $[\ldots]$

Ici, l'univers capitaliste.

Ici, l'univers prolétarien.

Et l'on voudrait qu'une révolution qui a ainsi bouleversé tous les partis politiques, ne laissant plus subsister dans le monde entier que deux classes en présence l'une de l'autre, ne soit pas une révolution de classe, la vôtre, ô travailleurs!

Allons donc!

Le 18 mars vous appartient tout entier, comme 89 appartient au Tiers Etat. Ou, plus exactement, le 18 mars est votre 89 - qui commence."

Des aspects particuliers de l'histoire de la Commune vont être approfondis par la suite. Un premier article, du 18 mars 1880 , de la main probablement de Lafargue, ${ }^{1}$ étudie la naissance du prolétariat qui est défini essentiellement par l'exploitation qu'il subit. Analysant brièvement 1848 et 1871, il constate les progrès accomplis: en 1848, le gouvernement a pu vaincre les ouvriers avec l'aide de la province; en 1871, malgré ses appels, Thiers n'a pas reçu cette aide et en conclusion, l'auteur prédit que:

1 Les articles, sauf exception, ne sont pas signés. 
«La prochaine Révolution trouvera debout le Prolétariat industriel de toutes les villes de France; elle trouvera debout le Prolétariat agricole qui grandit avec la concentration foncière; elle trouvera debout les paysans-propriétaires jugulés par la concurrence de la grande culture, par la concurrence étrangère. La prochaine Révolution embrassera toute la France..."

Démonstration reprise le 23 mai, sous le titre "Crescendo". Après avoir évoqué 1831 et 1848 , on montre l'élargissement que représenta la révolution de 1871: "Par la présence d'étrangers au sein de la Commune et au nombre de ses combattants, par l'absence de tout préjugé de patriotisme, par les côtés généreux de son programme, par ses conséquences, la Révolution du Dix-Huit Mars a le caractère d'un mouvement international." La conclusion insiste sur la nécessité d'élever encore les luttes jusqu'à la prochaine révolution: "Que sera la Commune à côté de la prochaine Révolution sociale? Il est permis de présumer qu'entre cette Révolution et la Commune, il y aura une aussi grande différence qu'entre la Commune et les mouvements qui l'ont précédée, si on songe surtout qu'entre elle et nous, qui avons pour mission de préparer la Révolution à venir, il y a,comme cela a déjà été écrit ici même, la différence des vagissements du nouveau-né à la parole humaine."

Pour la première fois, par l'intermédiaire de Lafargue sans doute, une explication directement "marxiste» est donnée et s'appuie expressément sur la Guerre civile de Marx, texte alors à peu près inconnu en France. Cette analyse paraît le 2 juin, sous le titre "La lutte des classes»: "La Commune de Paris fut une des plus éclatantes manifestations de la lutte des classes qui déchire la société capitaliste." A la différence de ce qui s'est passé en 1831, le prolétariat s'est emparé du pouvoir d'Etat. Fait nouveau, car jusqu'alors il ne luttait que sur le terrain économique. "C'est dans l'action politique que la classe ouvrière trouvera le moyen de résoudre les antagonismes sociaux." La Commune lui a permis de s'affirmer "sur le terrain politique». Le prolétariat n'était pas seul, d'où des erreurs, des tâtonnements qui lui furent fatals. La conclusion est messianique:

"Toutes les révolutions de ce siècle ont été faites par le peuple au profit de la Bourgeoisie. La Commune triomphante eût été faite par le peuple et pour le peuple. Et cependant, le lendemain de la victoire du Comité central, la classe ouvrière était à chercher quels seraient ses représentants et quelles seraient les mesures révolutionnaires qu'ils devaient prendre. La Révolution sociale que doit accomplir le Prolétariat de notre siècle, l'appropriation nationale des moyens de production, sera aussi grande que celle accomplie au siècle dernier par la Bourgeoisie.» 
Ainsi, le problème de l'Etat a-t-il été posé. La forme que doit prendre la future révolution est exposée le 11 août 1880 . Ici encore, la réflexion part d'une analyse de la Commune. Elle se différenciera de l'émeute (comme le soulignera un peu plus tard Engels, avec qui on ne peut manquer de faire le rapprochement):

«Avec les grandes armées modernes, la rapidité et la facilité de leurs mouvements de concentration, les révolutions ne peuvent plus être des coups de main parisiens, soudainement exécutés comme 1830 et 1848 . Le mouvement insurrectionnel du 18 mars, qui cependant fut vigoureusement soutenu par quelques villes de province, prouve mieux que tout raisonnement que la révolution prolétarienne doit soulever toute la masse ouvrière du pays, pour offrir quelque chance de succès. [...] La révolution prolétarienne ne sera ni locale, ni nationale, elle sera internationale ou elle ne sera pas."

Cet article se situe dans le cadre de la préparation du Congrès du Havre (16-22 novembre 1880) et vise essentiellement les anarchistes que compte encore le parti ouvrier. La conclusion annonce en quelque sorte le possibilisme:

«Les hommes du Parti ouvrier ne sont pas des phraseurs, croyant aveuglément aux incantations anarchistes qui n'effraient même plus les rédacteurs du Siècle; ils sont des hommes d'action qui pensent que pour terrasser l'Etat capitaliste, il ne faut pas l'ignorer et l'éviter, mais le combattre au jour le jour et s'en emparer pièce à pièce. On ne démantèle une forteresse que lorsqu'on l'a emportée d'assaut."

Ainsi, la Commune est-elle définie comme un mouvement prolétarien, résultat de toute une évolution du mouvement ouvrier, mais inadapté en somme aux conditions du moment en raison de son isolement. Ce mouvement portant en lui les germes de la future révolution, l'Egalité montre qu'il existait des débuts d'extension provinciale et de solidarité internationale: d'où l'article sur la province, le récit des réactions en Angleterre, en Belgique, Espagne, Italie, Russie, aux Etats-Unis (18 mars 1880).

La démonstration se situe aussi sur le plan théorique. Le proudhonisme fait l'objet des plus vives attaques. Le 14 avril, Lafargue certainement encore prend comme prétexte la lettre d'un cordonnier, partisan des coopératives, pour critiquer la politique des Tolain, Fribourg et Limousin essayant dans les années soixante d'introduire la question de la coopération dans les congrès de l'Internationale. Mais, il ne rejette pas l'idée que cette coopération "dans un milieu où 
l'appropriation capitaliste a été abolie» puisse "devenir une forme transitoire des sociétés humaines». Parallèlement, il critique l'ouvriérisme de ce lecteur et s'appuie sur l'exemple de la Commune. Le but, «l'émancipation de la classe ouvrière par la classe ouvrière ellemême", n'empêche nullement que des transfuges de la bourgeoisie ou des ouvriers "privilégiés", puissent combattre aux côtés des ouvriers. «Est-ce que Duval, Varlin, Flourens, Tony-Moilin ont été repoussés et insultés?»

La publication en feuilleton de la Misère de la Philosophie de Marx (7 avril, 14 avril, 5 mai) entre dans cette série d'attaques théoriques contre le proudhonisme, mais le 16 juin, en raison des multiples interruptions, le journal décide d'interrompre la publication et de faire paraître le texte en brochure. Il ne semble pas que celle-ci ait vu le jour. La publication du début de La Guerre civile, dans Le Socialiste de 1887, ne sera pas non plus poursuivie. Il faudra attendre 1900 pour la traduction (incomplète) de Charles Longuet sous le titre: La Commune de Paris.

L'appréciation de Claude Willard, que les guesdistes "n'analysent point l'expérience communarde et semblent toujours ignorer l'étude que Marx lui a consacrée»,1 parfaitement valable à partir de 1881, ne paraît pas fondée s'agissant de cette deuxième série de l'Egalité. On peut s'interroger sur les raisons de l'oubli dans lequel va tomber l'événement de 1871. Ici, au contraire, la lutte doctrinale, sous tous ses aspects, s'appuie sur des exemples et des enseignements de la Commune.

\section{Les conséquences de la Commune: la nécessité d'un programme}

La première série de l'Egalité du 18 novembre 1877 au 14 juillet 1878 , sous la direction de Guesde, mêlait toutes les orientations possibles des courants socialistes et libertaires. Quand débute la deuxième série, le 20 janvier 1880, l'orientation est plus précise. L'Egalité se situe dans la ligne du Congrès de Marseille, d'octobre 1879, qui «aiguille le mouvement ouvrier français sur une nouvelle voie, la voie collectiviste et révolutionnaire». ${ }^{2}$ La coalition de marxistes, d'anarchistes et de futurs possibilistes avait triomphé. Il s'agissait pour les amis de Guesde de créer un parti ouvrier révolutionnaire; la naissance d'un "organe collectiviste révolutionnaire» sous la devise générale «Liberté, solidarité, justice» constitue un des moyens de la lutte pour le programme

1 Claude Willard, Le mouvement socialiste en France (1893-1905), Les Guesdistes (Paris 1965), p. 196.

2 Claude Willard, Le mouvement socialiste en France, op. cit., p. 16. 
marxiste. Dans cette perspective, l'essentiel des articles est consacré aux éléments de ce programme. Mais, l'expérience de la Commune, invoquée souvent, y compris lors de la commémoration, répond aussi à ce but. La Commune est considérée comme une étape qu'il s'agit de dépasser, qui sera dépassée par la Révolution prolétarienne qui approche. Il lui manquait essentiellement un programme politique, économique et social. Tout en luttant contre le cléricalisme, les radicaux, la coopération, l'Egalité en lance les grandes idées et y intègre constamment ses analyses de l'expérience de la Commune. Le journal insiste sur l'aspect irréversible du processus engagé:

«La lutte de classe que nous poursuivons pour la suppression des classes dans l'instrument de travail gratuit et de travail obligatoire, exige un prolétariat conscient, organisé; ce qui est affaire de temps. Et jusque là, traître à lui-même et à la cause, quiconque, par une action prématurée, reculerait l'heure de l'action efficace. $[\ldots]$

Ces victimes de la plus grande guerre sociale, arrachées aux six pieds de terre qui les couvrent, à la chaux vive où vous pouviez les croire consumées, - et couronnées de fleurs, ne présagent pas de longs jours à votre domination épuisée!

Mais à qui la faute? Si proscripteurs que vous soyez, comment proscrire le souvenir?

Le sang est tiré; - il faut le boire. $\|^{1}$

Suit une longue rubrique sous le titre «Les lendemains de la Commune à l'étranger et en France», composée d'extraits de programmes de partis étrangers ou de déclarations d'intention; tous prolongent certaines expériences de la Commune et intéressent le futur programme du Parti ouvrier: rappel des programmes du parti démocrate socialiste allemand (Gotha, mai 1875), du Socialistic Labor Party des EtatsUnis (1877), des socialistes danois (1872), du parti socialiste belge (1877), du parti ouvrier espagnol (1879), de la fédération des Travailleurs suisses (1878), du programme nihiliste de Russie, du programme du Cercle socialiste de Milan (1877) et de la déclaration des CollectivistesRévolutionnaires à Marseille (1879). Ces textes sont très importants pour les rédacteurs de l'Egalité qui conçoivent la Commune, non comme modèle, mais comme première expérience de la prise du pouvoir politique par le prolétariat. Le 2 juin, Lafargue encore une fois certainement souligne cette "possession des pouvoirs de l'Etat par les représentants du Prolétariat, conséquence du mouvement communaliste». La répression, pour l'auteur, était chose normale.

1 Numéro des 23-26 mai 1880. 
Ainsi, la Commune est-elle surtout conçue comme susceptible de donner l'exemple de la nécessité de la prise du pouvoir politique par les travailleurs; elle n'était pas une expérience socialiste, mais une étape vers celle-ci, une transition nécessaire. D'où la publication, le 30 juin, du projet de programme électoral des travailleurs socialistes, qui occupe toute la première page du journal avec des rappels directs de l'œuvre de la Commune. Suit le commentaire: "Il contribue plus que toute argumentation théorique à le [le prolétariat] libérer des dernières illusions réformistes et à le convaincre de l'impossibilité de faire l'économie d'un 89 ouvrier."

L'Egalité insiste davantage sur les facteurs de l'événement qui peuvent inciter à la réflexion dans la bataille d'idées que sur l'aspect émotionnel. L'absence de programme de la Commune, conçue comme une étape décisive, l'a rendue impuissante. Sa défaite est due essentiellement au fait qu'elle s'attaquait, pour la première fois, au pouvoir politique. Le prolétariat, qui était le noyau principal de ce mouvement doit définir un programme collectiviste et ne plus se contenter, exclusivement, de luttes sur le terrain économique. Il doit aussi combattre pour des réformes partielles, autant d'étapes vers le socialisme. Dernier aspect qui sera vivement combattu dans la troisième Egalité, à partir du 11 décembre 1881, dirigée contre les amis de Brousse. Fait significatif, dans la période mars-mai 1882, l'Egalité ne consacre qu'un seul article de Guesde à la Commune. Etait-elle oubliée en tant qu'expérience pratique permettant la réflexion? Il est vrai que cette fois, ce sont les adversaires des Guesdistes qui sollicitent son héritage! A partir de cette date tout en la défendant quand il le faut, ils se contenteront de réflexions commémoratives. 